\title{
PHONON SCATTERING DUE TO DEEP ACCEPTORS IN SEMICONDUCTORS
}

\author{
A. de Combarieu ${ }^{+}$and K. Lassmann ${ }^{++}$ \\ +S.B.T., CENG, BP.85, 38041 Grenoble, France \\ ${ }^{++}$Physik. Institut, Teil 1, 7 Stuttgart 80 , Germany
}

We have measured the magnetothermal conductivity in GaAs(Mn)

$\left[3.8 \times 10^{18} \mathrm{~cm}^{-3}\right]$ and $\mathrm{Si}(\mathrm{In})\left[5 \times 10^{15} \mathrm{~cm}^{-3}\right]$ for temperatures between $1.4 \mathrm{~K}$ and $90 \mathrm{~K}$ at magnetic fields up to $8 \mathrm{~T}$. In both cases the dopants are deep acceptors with binding energy much larger $(110 \mathrm{meV}$ and $165 \mathrm{meV}$ respectively) than given by the effective mass theory $(\sim 35 \mathrm{meV})$. There is a double interest in such systems: First, an excited level $3 \mathrm{meV}$ ( $4.2 \mathrm{meV}$ ) above the acceptor ground state has been concluded from ultrasonic measurements $/ 1 / / 2 /$. Such an excited state might be connected with a Jahn-Teller effect of these deeper acceptors and should be seen by resonant phonon scattering in thermal conductivity. Second, an anomalous behavior of the magnetothermal conductivity has been found for shallow acceptors in Ge (but not in Si) /3/ making comparison with systems with different g-factors desirable. The $g$-factors of acceptors in GaAs are roughly three times, the $\mathrm{g}$-factor of $\mathrm{Si}(\mathrm{In})$ about 0.6 times that of $\mathrm{Si}(\mathrm{B})$.

In zero magnetic field in both cases we see a strong reduction. of the thermal conductivity at low temperatures as compared to the pure material scaling well in concentration with the reduction found for shallow acceptors; furthermore, a distinct dip is seen at about $23 \mathrm{~K}$ for $\mathrm{Si}(\mathrm{In})$ (Fig. 1) and at about $12 \mathrm{~K}$ for GaAs(Mn) (Fig. 2). The same dip has been found for GaAs(Mn) by Holland /4/ at concentrations lower than ours. The maximum thermal conductivity for $S i(I n)$ is reduced due to about $10^{18} \mathrm{~cm}^{3}$ oxygen contained in the sample $/ 2 / / 5 /$. The dependence on magnetic field is analogous to that found by Chal$l i s$ and Halbo $/ 3 /$ for $S i(B)$ : The relative thermal conductivity $\boldsymbol{x}_{B} / \boldsymbol{x}_{0}$ for $\mathrm{GaAs}(\mathrm{Mn})$ first falls to a minimum value of about 0.6 (due to an enlarged resonant scattering) and then rises rapidly (Fig. 3). However, this rise tends to saturate at the highest fields to a value about ten times below that of the pure crystal, which may be due to 


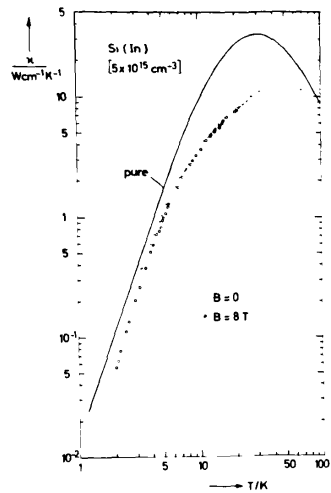

Fig. 1 Thermal conductivity in $\mathrm{Si}(\mathrm{In})$.

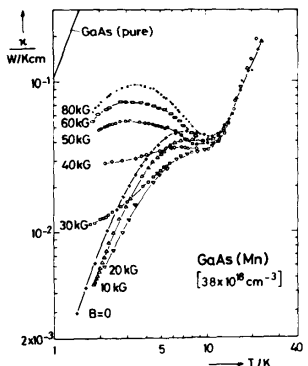

Fig.2 Thermal conductivity in $\mathrm{GaAs}(\mathrm{Mn})$ at several magnetic field strengths.

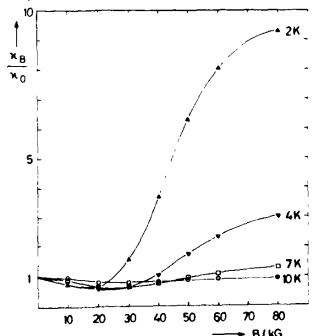

Fig. 3 Relative thermal conductivity $x_{B} / x_{0}$ in $\mathrm{GaAs}(\mathrm{Mn})$ as a function of magnetic field at various temperatures. 
the low frequency part of the resonance scattering associated with the dip at $12 \mathrm{~K}$. As is expected from the small $\mathrm{g}$-factor, the variation of $\alpha_{B}$ /of for $S i$ (In) is slow; that is, only a reduction is seen attaining $\mathrm{a}$ minimum value of 0.8 at $2 \mathrm{~K}$ and $8 \mathrm{~T}$. Thus, in both cases the magnetic field dependence does not show the "anomaly" found for shallow acceptors in Ge. However, the variation of $\alpha_{B} / x_{0}$ for $\mathrm{Si}$ (In) seems to be somewhat slower than can be accounted for by the $\mathrm{g}$-factor and, in the case of $\operatorname{GaAs}(\mathrm{Mn})$, the minima of $x_{B} / x_{0}$ at different temperatures do not scale very well with $B / T$, but there is a part $\alpha(B / T)^{2}$.

For an analysis of $x_{0}$ we applied a modified model for the acceptor ground state: A distribution of small splittings $\delta$ (determining the low temperature part of the scattering) and an excited level $\Delta$ above these split levels. The extended nature of the acceptor wave function, reducing the interaction with short wavelength phonons, was taken into account (Bohr-radius $10 \AA$ for $\operatorname{GaAs}(\mathrm{Mn})$ and $7.4 \AA$ for $\mathrm{Si}(\mathrm{In}))$. The formula for resonance fluorescence scattering in the form given by Suzuki and Mikoshiba /6/ was applied. The assumption $\delta \ll k T$ made for ease of calculation underestimates the scattering at the lowest temperatures. A good fit following the acute variation of $\boldsymbol{x}_{0}$ in the dip region especially for GaAs(Mn) was not possible. In both cases it was necessary to take a smaller Bohr radius for the excited level ( $3 \AA$ for $\operatorname{GaAs}(M n))$. The best values for $x_{0}$ thus obtained are $5 \mathrm{meV}$ for $\mathrm{Si}(\mathrm{In})$ and $3 \mathrm{meV}$ for $\mathrm{GaAs}(\mathrm{Mn})$. This has to be compared with the values analyzed from ultrasonic measurements. A more direct determination of these energies with quasimonochromatic phonons $/ 7 /$ is desirable.

One of us (K. L.) gratefully appreciates the cordial hospitality of the group of Service Basses Temperatures, CEN Grenoble.

\section{References:}

/1/ Hp. Schad and K. Labmann, Conf. on Microwave Acoustics, Lancaster $(74)$ p. 191

$/ 2 / \mathrm{Hp}$. Schad and $\mathrm{K}$. Laßmann, these Proceedings

$/ 3 /$ L. J. Challis and L. Halbo, Phys. Rev. Lett. 28, 816 (72)

$14 /$ M. G. Holland, Proc. $7^{\text {th }}$ Int. Conf. Semiconductors, Paris (64), p. 713

15/ M. G. Holland, Proc. Int. Conf. Semiconductors, Prague (60) p. 633

/6/ K. Suzuki and N. Mikoshiba, J. Phys. Soc. Jap. 3144 (71)

$/ 7 /$ W. Eisenmenger, these Proceedings. 